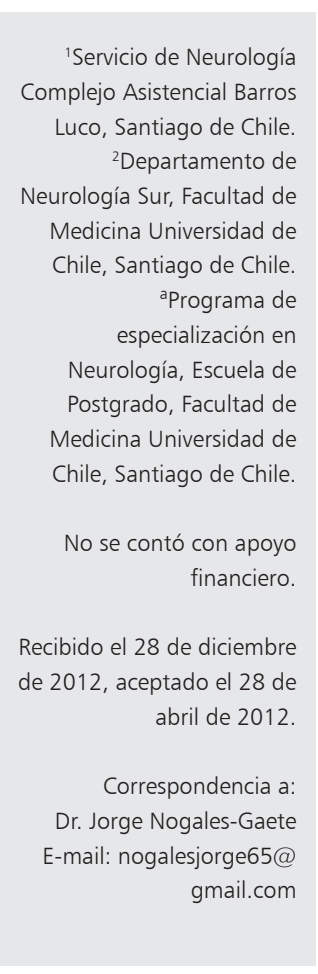

\section{Información médica a pacientes y familiares: aspectos clínicos, éticos y legales} JORGE NOGALES-GAETE ${ }^{1,2}$, PAOLA VARGAS-SILVA ${ }^{1,2, a}$, IVÁN VIDAL-CAÑAS ${ }^{1,2, a}$

\section{Clinical, ethical and legal issues on medical information to patients and relatives}

\begin{abstract}
Providing information to patients and relatives (IPAR) is a clinical, ethical and legal need. IPAR is inherent to the medical team work as diagnostic and therapeutic procedures are. In some cases, it is a key step for patient's recovery. From an ethical point of view, the patient is not only the subject of the medical work but also its main owner and who must, previously informed, decide about options which would directly affect him. After the promulgation of a special law in Chile, this prerogative became a legal right. This paper proposes an empirically developed model or protocol that should be used to inform hospitalized patients and their relatives about their disease, in a pertinent way. Considering that IPAR is a skill that should be learned and practiced, the written protocol is provided to neurology residents as supporting material. Although IPAR protocols are completely justified, they have not been evaluated in terms of efficiency of communication, user satisfaction, patient and relatives reassurance and clinical impact. Therefore, they require a prospective validation.
\end{abstract}

(Rev Med Chile 2013; 141: 1190-1196).

Key words: Ethics, medical; Information seeking behavior; Physician-patient relations.

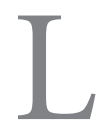

a información al paciente y familiares (IPYF) es un valor fundamental de la relación médico-paciente, cuyo sustento clínico, ético, legal y deontológico ha ido variando en relevancia, acorde a los cambios culturales de la sociedad $^{1-3}$. La IPYF es una actividad tan propia y cotidiana del quehacer clínico como lo es el proceso diagnóstico o de tratamiento, pudiendo en algunos casos ser la pieza central de la recuperación del paciente o un elemento trazador para evaluar la calidad de la atención ${ }^{4}$. La adecuada comunicación también ha sido correlacionada positivamente, con un mejor diagnóstico, menos juicios por mala práctica, mejor adherencia al tratamiento, mayor satisfacción usuaria y mejor desenlace ${ }^{5,6}$. En lo ético, el paciente no sólo es el sujeto y usuario del acto médico, sino su principal dueño y quien debe decidir de manera informada sobre las opciones de estudio y tratamiento que le afectarán directamente ${ }^{4}$. En Chile, la promulgación de la ley 20.584, culmina un desarrollo legislativo ${ }^{4,7-9}$ sobre el marco de los "derechos y deberes que tienen las personas en relación con acciones vinculadas a su atención en salud", definiendo en el Artículo $8^{\circ}$ algunas características de la información que deben entregar los prestadores profesionales e institucionales. De acuerdo con este artículo la IPYF debe ser suficiente, oportuna, veraz y comprensible, ya sea en forma visual, verbal o por escrito, respecto de los siguientes elementos: a) Las atenciones de salud o tipos de acciones de salud que el prestador respectivo ofrece o tiene disponible y los mecanismos a través de los cuales se puede acceder a dichas prestaciones, así como el valor de las mismas; b) Las condiciones previsionales de salud requeridas 
para su atención, los antecedentes o documentos solicitados y los trámites necesarios para obtener la atención; c) Las condiciones y obligaciones contempladas en sus reglamentos internos que las personas deberán cumplir mientras se encuentren al interior de los establecimientos asistenciales?.

El presente documento propone a discusión de la comunidad clínica un modelo o protocolo diseñado empíricamente, para informar a enfermos hospitalizados y sus familiares, considerando nuestra experiencia y algunas publicaciones centrales al respecto ${ }^{10-16}$. Este protocolo lo hemos desarrollado para uso en el Servicio de Neurología del Hospital Barros Luco, como material de apoyo y formación de los residentes que se especializan en neurología, considerando el tema IPYF como una destreza clínica susceptible de capacitación y ejercitación ${ }^{6}$.

Asumimos que la buena relación médicopaciente o médico-familiares es única, irrepetible y diversa caso a caso, por lo que este modelo no busca reemplazar el arte y el profesionalismo clínico, se ha dividido el proceso IPYF en puntos que se presentan en la Tabla 1. Hemos optado por una presentación sumaria, casi a la manera de un listado instructivo, siendo fieles a su texto original.

\section{Protocolo de información médica a pacientes y familiares}

\section{Preparación de la entrevista}

\section{Organización de los antecedentes}

La organización global de los antecedentes busca definir: quiénes serán informados, cuál es la situación de salud en que se encuentra el paciente, qué sabe éste de su problema y de esta categoría de problemas, qué y cuánto desea saber y en qué condiciones está de conocer de su problema y qué considera el médico o el equipo médico que debería saber el paciente, para una mejor gestión de sus decisiones sobre la enfermedad y prioridades de vida.

Es básico conocer en forma detallada la historia del enfermo, incluyendo aspectos: antropológicos, sociales, familiares y clínicos. El sentido e impacto de la enfermedad en el individuo varía según estos elementos e incide en cómo debe ser informado el paciente y sus familiares o representantes.

La adquisición de un método en la IPYF es tan necesaria y útil como lo es en el aprendizaje de configurar una anamnesis o un examen físico; quien tiene una organización conceptual tendrá mejor capacidad de improvisación y adaptación en situaciones de urgencia o especiales.

Para evitar omisiones en la entrega de la información es útil preparar una lista de chequeo personal (checklist) de los contenidos.

\section{Organización de la interacción}

Si el paciente está consciente y sin deterioro cognitivo relevante, deberá preguntársele si existe algún tipo de restricción de su parte acerca de la información que se entregará a los familiares. Esto acoge no sólo un precepto ético, sino también una garantía constitucional relacionada con el respeto a la vida privada y la honra de la persona ${ }^{17}$.

Si el paciente presenta una alteración de conciencia o cognitiva relevante se debe informar con prudencia a los familiares directos cautelando su intimidad. Para este fin, es útil establecer un apoderado o familiar responsable, acreditado formalmente en ese rol según corresponda por la voluntad del paciente, la familia o las reglas de la institución. Esto reduce conflictos que pueden producirse por múltiples interlocutores con igual número de versiones

Tabla 1. Protocolo de información médica a pacientes y familiares

I. Preparación de la entrevista Organización de los antecedentes Organización de la interacción

II. Convirtiendo un acto pertinente y cotidiano en un signo de acogida

Necesidades y percepciones del paciente

Estructura de un resumen y conclusiones

El momento y el escenario, lo mejor dentro de lo posible

La identificación de los interlocutores

Receptividad a las inquietudes del paciente y su familia Consentimiento informado

III. Contenidos básicos a tratar

Situación presente

Proyección de futuro

IV. Particularidades para la entrega de malas noticias

Sobre el informante

Sobre el escenario

Sobre el paciente

Sobre la modalidad de entrega

Sobre el contenido 
y facilita la agenda de los momentos de encuentro entre el médico tratante y la familia. Como contraparte, el médico tratante debe estar dispuesto e informado para representar a todo el equipo, en los aspectos generales. Esto permite una planificación oportuna y adecuada de la interrelación entre el equipo profesional y la familia.

Un aspecto muy complejo que trasciende de lo clínico a lo ético, es valorar la capacidad del paciente para recibir y usar la información cuando éste presenta deterioro cognitivo. El paradigma de esta situación es la comunicación del diagnóstico de la enfermedad de Alzheimer al paciente ${ }^{18}$. En este escenario, creemos que la familia juega un rol fundamental en permitirnos conocer la voluntad del paciente, para cautelar con armonía sus derechos y necesidades clínicas.

No es pertinente proporcionar información a personas que no tienen relación directa con el enfermo ${ }^{17}$. Si no se trata del apoderado y la situación se plantea con un familiar cercano, se debe explicar en forma amable que existe un apoderado a quien se le entregará la información. No corresponde entregar información a familiares no directos o a través de vía telefónica ${ }^{17}$. Facilita la comunicación médico paciente o familia, definir una instancia diaria de contacto para escuchar inquietudes, constituyendo además una ayuda a la buena relación institucional con los familiares, el indicar el horario en el cual puede ser visitado el paciente.

\section{Convirtiendo un acto pertinente y cotidiano en un signo de acogida}

\section{Necesidades y percepciones del paciente}

La comunicación es efectiva si el receptor logra captar el mensaje; la forma de realizarlo es usuario dependiente, por lo que debe considerarse el nivel cultural y adaptarse a éste para lograr un entendimiento adecuado. Del mismo modo la información es útil sólo cuando es oportuna, de allí la caracterización de la ley respecto de la IPYF como: suficiente, oportuna, veraz y comprensible 9 .

Los pacientes y familiares valoran positivamente un lenguaje simple, con ausencia de metáforas innecesarias. La información debe armonizar la precisión con la integralidad ${ }^{13,19,20}$. En general no se requiere una explicación detallada de toda la enfermedad, sino más bien dar una información relacionada con las inquietudes particulares del paciente, considerando el plan de manejo pro- puesto, con una estimación de tiempo, costo y eventuales protecciones sociales especiales relacionadas con la enfermedad, como sucede en Chile con el sistema de garantías nacionales GES o la ley de urgencia. Finalmente, es importante plantear el pronóstico, para que los familiares estén preparados para eventuales malos escenarios, sean estos vitales o de discapacidad ${ }^{13,21}$.

Es importante considerar que él o los familiares, habitualmente se encuentran emocionalmente involucrados en el problema del paciente y que, por su dolor y ansiedad, pueden estar poco receptivos a la información o incluso manifestar molestia, ya sea por la atención recibida, por las dificultades de acceso o por esperas prolongadas, lo que puede conducir a una conducta incluso agresiva. Es necesario buscar el tiempo y actitud para trabajar este aspecto ${ }^{10,15}$.

Es recomendable dirigirse al paciente, apoderado o familiar de manera formal, respetuosa y cordial. La empatía y cordialidad deben tener coherencia no sólo en el discurso, sino también en las claves del lenguaje no verbal y en las actitudes ${ }^{21}$.

\section{Estructura de un resumen y conclusiones}

Atendiendo al objetivo y destinatario de la información, es importante recapitular y reiterar lo fundamental, guiado por la pregunta ¿y Ud. que entendió respecto del aspecto XX? La conclusión debe ser coherente con el plan de manejo acordado. El resumir y concluir no sólo es central, sino que puede ser complejo y de alto impacto, por lo que debe atenderse con prolijidad ${ }^{11,15,22}$.

\section{El momento y el escenario, lo mejor dentro de lo posible}

Es necesario elegir el momento y lugar más adecuado para IPYF, considerando: la necesidad del paciente, si se trata o no de una urgencia médica, la realidad de los tiempos disponibles y las limitaciones de los espacios físicos disponibles para esta función. Siempre es importante cautelar la discreción y privacidad, incluso si las noticias son buenas.

Es importante tener presente que la primera entrevista puede ser la única oportunidad de contacto con algún familiar, de modo que es necesario revisar si existen aspectos de importancia pendientes que consultar, especialmente aquellos que determinan una conducta de limitación de esfuerzo terapéutico en caso de agravamiento. 


\section{La identificación de los interlocutores}

$\mathrm{Al}$ iniciar la entrevista, corresponde saludar e identificarse mencionando el nombre, rol en el equipo y especialidad, del mismo modo que se debe revisar y confirmar la identificación del paciente, con nombre completo y cama o habitación donde está ubicado. De igual manera se debe identificar, cuando corresponde, al "apoderado" o "responsable legal" para una posible toma de decisión, en que fuera necesario apoyar o representar al paciente en esa situación. Consignar su nombre y relación parental en ficha médica, así como información entregada.

\section{Receptividad a las inquietudes del paciente y su familia}

Muchas veces el enfermo o su familia preguntan acerca de otros posibles tratamientos, incluyendo medicina alternativa y complementaria; si está en conocimiento de lo que le preguntan, responda considerando no sólo la evidencia sobre la indicación y la eficacia de la alternativa propuesta, sino que también contraindicaciones, efectos adversos, y accesibilidad. Cuando corresponda, se debe explicitar los niveles de incerteza correspondientes ${ }^{11}$.

\section{Consentimiento informado}

En el caso de necesitar un consentimiento informado para un determinado procedimiento se deberá entregar toda la información acerca de riesgos y beneficios. En ausencia de evidencia formal, es válido orientar y recomendar de acuerdo a informaciones obtenidas en reuniones clínicas, revisiones bibliográficas de casos similares y experiencia del propio servicio, advirtiendo sobre la fuente ${ }^{3}$.

La ley 20.584 sobre derechos y deberes de los pacientes entre sus múltiples aspectos instruye informar sobre: las instancias, personas y mecanismos donde averiguar sobre los costos de la atención, establece el derecho a rechazar; exámenes, terapias y a solicitar el alta según su voluntad y responsabilidad. Del mismo modo explicita condiciones que requieren formalmente de consentimiento informado: exámenes, procedimientos, intervenciones quirúrgicas, ingreso a protocolos de investigación científica, registro clínico de imágenes, sea por motivos de control evolutivo o de docencia?.

Se presume que la persona ha recibido la información pertinente para determinado consentimiento, cuando hay constancia de su firma, o del apoderado cuando corresponda por edad o limitación, en un documento explicativo y explicito del procedimiento, tratamiento o autorización de uso de información clínica aludida ${ }^{9}$. La ley 20.584, en su artículo 14, establece que la modalidad de consentimiento debe ser escrita para procedimientos, intervenciones y tratamientos con riesgo y en el artículo 20 lo explicita para cualquier forma de investigación científica biomédica. En la ley no se comenta sobre el formato ni situaciones donde sea pertinente la modalidad verbal.

\section{Contenidos básicos a tratar}

\section{Situación presente}

Es necesario establecer la situación clínica del paciente en forma actualizada, incluyendo grados de mejoría o deterioro. Si no existe definición del diagnóstico, es pertinente informar la naturaleza de la dificultad y los caminos que se usarán para descartar o confirmar la sospecha clínica. Es importante para la organización de las decisiones del paciente y su familia, comentar las eventuales complicaciones a corto, mediano y largo plazo, que suelen presentarse, mencionando factores de riesgo.

\section{Proyección de futuro}

Desde el inicio de la relación médico tratantefamilia, es importante conocer cuáles son las condiciones socio económicas de la familia para recibir y cuidar al paciente en el momento del alta, identificar al familiar que cuidará al paciente en su casa y cuando sea pertinente conectarlo con la enfermera y kinesiólogo o con el programa integral de educación de preparación de los cuidados post alta. En situaciones de discapacidad para algunas actividades, es conveniente instruir al familiar respecto de nuevas necesidades, cuidados y de las competencias necesarias para asistir al paciente. $\mathrm{Si}$ el alta es inminente, es necesario recapitular la educación sobre la prevención de factores de riesgo.

\section{Particularidades para la entrega de "malas noticias"}

Se definen como malas noticias a las que producen una severa alteración negativa en las expectativas del porvenir del paciente. Para esta 
situación es válido el mismo marco ya expuesto, pero con énfasis en algunos puntos ${ }^{12,13,16,24}$.

\section{Sobre el informante}

El médico debe estar preparado técnica y psicológicamente para resolver dudas en forma precisa; las vacilaciones y contradicciones, generan no sólo más dudas, sino una dolorosa incertidumbre y la pérdida de confianza en los interlocutores.

\section{Sobre el escenario}

Se debe intentar brindar la información al paciente con la privacidad que corresponde. $\mathrm{Mu}-$ chas veces el entregar malas noticias a pacientes o en relación a pacientes en ambientes abiertos e inadecuados, puede causar un efecto deletéreo en el paciente o en sus familiares, o bien, puede contribuir a que no se aclaren en forma precisa las dudas derivadas de su patología y en su entorno ${ }^{10,13}$.

\section{Sobre el paciente}

Importante es indagar qué es lo que conoce el paciente o familiares de su enfermedad y qué es lo que desean saber. Si bien todo enfermo tiene derecho a conocer su real estado y pronóstico, existen numerosos estudios y reportes en la literatura médica en la cual se demuestra que un porcentaje no menor de pacientes, no desean informarse de las malas noticias que conlleva su enfermedad ${ }^{13,19}$. Encuestas realizadas a pacientes con ciertas patologías de mal pronóstico como neoplasias malignas o esclerosis múltiple muestran que hasta $28 \%$ de los enfermos no desean informarse respecto del mal pronóstico de su enfermedad y desearían que dicha información le fuera brindada de forma íntegra y exclusiva a sus familiares ${ }^{13,19}$.

\section{Sobre la modalidad de entrega}

Debe usarse un lenguaje simple. Puede incluso utilizarse material complementario audio-visual cuando sea necesario ${ }^{11,16}$. Además de la pulcritud técnica se debe actuar con el tacto, sutileza, empatía y afectividad que la situación amerita. Una mala noticia brindada de mala forma puede ser tanto o más deletérea que la información en sí.

Es muy importante considerar que la veracidad tiene niveles de extensión y detalle, por lo que se requiere una prudente consideración de las necesidades terapéuticas y vitales para graduar este aspecto, la comunicación de la verdad debe asumirse como un objetivo de ayuda al individuo y no destructora de éste (primum non nocere). El tratante debe reflexionar sobre el momento y capacidad del paciente para recibir la verdad; evitando consecuencias autodestructivas. Para esto es importante considerar su etapa en el ciclo vital, el temple y la presencia de situaciones personales no resueltas.

\section{Sobre el contenido}

$\mathrm{Al}$ organizar la entrega considerando: lo necesario, lo importante, lo requerido por el paciente, lo definido por el marco legal e institucional, y lo que el médico requiera para alguna acción, debe existir coherencia entre lo entregado y el objetivo o uso que se dará a la información. Es útil incluir información (cuando exista) de la continuidad de la atención y opciones de manejo, en los diferentes escenarios, para reducir la sensación de desamparo que puede generar la proyección evolutiva.

Es importante determinar a priori, en casos de pacientes muy graves o terminales, eventos tales como limitación del esfuerzo terapéutico y la reanimación cardio-respiratoria. Muchas veces existen directrices anticipadas que el mismo paciente ha realizado, por ejemplo, una declaración de voluntad por escrito o bien el testimonio de sus cercanos. Esto es de gran ayuda en el momento de concordar decisiones.

La ley sobre derechos y deberes de los pacientes establece la posibilidad de recibir visitas de un guía espiritual, esto si bien es pertinente en todas las condiciones, en las situaciones de enfermedad grave, con riesgo vital, es aun más relevante ${ }^{9}$.

\section{Reflexión final}

Aun cuando no existen dudas de la importancia que tiene la IPYF en el acto médico, es necesario considerar las dificultades que existen para evaluarla ${ }^{20,25,26}$, ya sea en términos de resultados focales como: análisis de estructura de la comunicación, eficacia comunicacional, satisfacción usuaria, tranquilidad del paciente y su familia, impacto clínico, calidad de vida o bien, ante resultados globales o integrales. Existen autores que tienen posturas entusiastas sobre los protocolos y la capacitación en IPYF ${ }^{27-29}$, otros mantienen una postura de activa evaluación sobre sus posibles beneficios ${ }^{30} \mathrm{y}$ algunos tienen actitudes muy críticas, respecto de las guías y protocolos de IPYF ${ }^{31}$. Lamentablemente, la evidencia sustentada en estudios metodológicamente correctos es escasa y poco categórica ${ }^{11,25,32,33}$. 
Por otro lado, las percepciones positivas de la comunicación, no siempre son concordantes entre el médico y el paciente $e^{5,34,35}$, así como tampoco existe acuerdo en el binomio aludido sobre el equilibrio o énfasis que debe existir entre las actitudes y afectividad de la entrega versus la pureza conceptual técnica del contenido ${ }^{36}$. Respecto de la capacitación en las destrezas de la comunicación, aunque existe cierto acuerdo de que la IPYF es susceptible de mejorarse con iniciativas de formación, existe menos acuerdo sobre cómo deben ser éstas en cuanto a contenido, extensión, continuidad y $\operatorname{costo}^{37-39}$. Por esto, hasta que no dispongamos de estudios metodológicamente adecuados, con herramientas acreditadas, debemos ponderar con prudencia estas propuestas e iniciativas aunque sean bien intencionadas y representen el juicio de algunos clínicos con fundada experiencia.

\section{Referencias}

1. Rosselot E. Aspectos bioéticos en la Reforma de Atención de Salud en Chile. II. Discriminación, libre elección y consentimiento informado. Rev Med Chile 2003; 131: 1329-36.

2. Navarro M, Gabriele G, Jovell A. Los derechos del paciente en perspectiva. Aten Primaria 2008; 40 (7): 367-9.

3. Carvallo A. Médicos y profesionalismo. Pacientes e información. Rev Med Chile 2005; 133: 253-8.

4. Rosselot E. Derechos del paciente, en el marco de la calidad de la atención médica. Rev Med Chile 2000; 128: 904-10.

5. Ruiz-Moral R, Pérez E, Perula de Torres L, De la Torre J. Physician-patient communication: a study on the observed behaviours of specialty physicians and the ways their patients perceive them. Patient Educ Couns 2006; 64 (1-3): 242-8.

6. Cockburn J, Walters W. Communication between doctors and patients. Curr Obstet Gynaecol 1999; 9: 34-40.

7. Bórquez G, Raineri G, Bravo M. La evaluación de la «capacidad de la persona»: en la práctica actual y en el contexto del consentimiento informado. Rev Med Chile 2004; 132: 1243-8.

8. Arenas J, Trucco C. Información y consentimiento en la práctica urológica: revisión de la doctrina, legislación y jurisprudencia. Rev Chil Urol 2006; 71 (1): 9-13.

9. Ley 20.584. Regula los derechos y deberes que tienen las personas en relación con acciones vinculadas a su atención de salud. Ministerio de Salud. República de Chile 2012. Disponible en: http://www.leychile.cl/Navegar?id
Norma $=1039348 \&$ idVersion $=2012-10-01$ [Consultado el 12 de diciembre de 2012].

10. Baile W, Buckman R, Lenzi R, Glober E, Beale E, Kudelka A. SPIKES-A Six-Step Protocol for Delivering Bad News: Application to the Patient with Cancer. Oncologist 2000; 5 (4): 302-11.

11. Epstein R, Alper B, Quill T. Communicating evidence for participatory decision making. JAMA 2004; 291: 235966.

12. Narayanan V, Bista B, Koshy C. 'BREAKS' protocol for breaking bad news. Indian J Palliat Care 2010; 16 (2): 61-5.

13. Romero-García K, Cabrera-Gómez J. Modelo de comunicación de malas noticias a personas con esclerosis múltiple: retos de la ética clínica. En Arriagada C, Nogales-Gaete J, Editores, Esclerosis Múltiple: Una mirada Ibero-panamericana, Segunda Edición. New York, USA: Demos Medical Pub 2008. p. 927-33.

14. Vandekieft G. Breaking bad news. Am Fam Physician 2001; 64: 1975-8.

15. Simón P, Barrio I, Sánchez C, Tamayo M, Molina A, Suess A, et al. Satisfacción de los pacientes con el proceso de información, consentimiento y toma de decisiones durante hospitalización. An Sist Sanit Navar 2007; 30 (2): 191-8

16. Rabow M, McPhee S. Beyond breaking bad news: how to help patients who suffer West.J Med 1999; 171 (4): 260-3.

17. Ley 19.628. Sobre la protección de la vida privada. Proyecto de ley sobre la protección de los datos de carácter personal. Ministerio Secretaria General de la Presidencia. República de Chile 1999. Disponible en: http://www. leychile.cl/Navegar?idNorma=141599 [Consultado el 12 de diciembre de 2012].

18. Fuentes P, Prato J. Comunicación diagnóstica en enfermedad de Alzheimer. Reflexión y propuesta. Rev Med Chile 2012; 140: 811-7.

19. Nogales-Collao A, De la Huerta E, Nogales-Gaete J. Esclerosis Múltiple: Experiencias y Percepciones del Paciente, sobre el Impacto de la Enfermedad en su Vida. En Arriagada C, Nogales-Gaete J, Editores, Esclerosis Múltiple: Una mirada Ibero-panamericana, Segunda Edición. New York, USA: Demos Medical Pub 2008. p. 957-5.

20. Wills C, Holmes-Rovner M. Patient comprehension of information for shared treatment decision making: state of the art and future directions. Patient Educ Couns. 2003; 50 (3): 285-90.

21. DiMatteo R, Taranta A, Friedman H, Prince L. Predicting Patient Satisfaction from Physicians' Nonverbal Communication Skills. Med Care 1980; (4): 376-87. 
22. Quilligan S, Silverman J. The skill of summary in clinician-patient communication: A case study. Patient Educ Couns 2012; 86 (3): 354-9.

23. Retamales A, Cardemil G. Beneficios de la autonomía y consentimiento informado: Ejemplo de los Testigos de Jehová. Rev Med Chile 2009; 137: 1388-94.

24. Fallowfield L, Jenkins V. Communicating sad, bad, and difficult news in medicine. Lancet 2004; 363: 312-9.

25. Grunfeld E, Folkes A, Urquhart R. Do available questionnaires measure the communication factors that patients and families consider important at end of life? J Clin Oncol 2008; 26 (23): 3874-8.

26. Cegala D, Lenzmeier S. Physician communication skills training: a review of theoretical backgrounds, objectives and skills. Med Educ 2002; 36 (11): 1004-16.

27. Hahn S, Friedman D, Quigley H, Kotak S, Kim E, Onofrey M, et al. Effect of patient-centered communication training on discussion and detection of nonadherence in glaucoma. Ophthalmology 2010; 117: 1339-47.

28. Verhoeven V, Avonts D, Vermeire E, Debaene L, Van Royen P. A short educational intervention on communication skills improves the quality of screening for Chlamydia in GPs in Belgium: a cluster randomised controlled trial. Patient Educ Couns 2005; 57 (1): 101-5.

29. Broers S, Smets E, Bindels P, Evertsz F, Calff M, De Haes $\mathrm{H}$. Training general practitioners in behavior change counseling to improve asthma medication adherence. Patient Educ Couns 2005; 58 (3): 279-87.

30. Parry R. Are interventions to enhance communication performance in allied health professionals effective, and how should they be delivered? Direct and indirect evidence. Patient Educ Couns 2008; 73 (2): 186-95.

31. Salmon P, Young B. Creativity in clinical communica- tion: from communication skills to skilled communication. Med Educ 2011; 45 (3): 217-26.

32. Bazian Ltd. The effects of education on patient adherence to medication. Evid base Healthc Publ Health 2005; 9: 398-404.

33. Moore P, Wilkinson S, Rivera S. Communication skills training for health care professionals working with cancer patients, their families and/or carers. Cochrane Database Syst Rev 2004; (2): CD003751.

34. Kenny D, Veldhuijzen W, Van der Weijden T, LeBlanc A, Lockyer J, Le' Gare F, el al. Interpersonal perception in the context of doctor-patient relationships: A dyadic analysis of doctor-patient communication. Sos Sci Med 2010; 70 (5): 763-8.

35. Van den Brink-Muinen A, Van Dulmen A. Do our talks with patients meet their expectations? J Fam Pract 2007; 56 (7): 559-68.

36. Beach M, Roter D, Wang N, Duggan P, Cooper L. Are physicians' attitudes of respect accurately perceived by patients and associated with more positive communication behaviors? Patient Educ Couns 2006; 62 (3): 347-54.

37. Berkhof M, Van Rijssen J, Schellart A, Anema J, Van der Beek A. Effective training strategies for teaching communication skills to physicians: An overview of systematic reviews. Patient Educ Couns 2011; 84 (2): 152-62.

38. Veldhuijzen W, Ramc P, Van der Weijden T, Van der Vleuten C. Communication guidelines as a learning tool: An exploration of user preferences in general practice. Patient Educ Couns 2012; pii: S0738-3991 (12) 00389-8.

39. Barth J, Lannen P. Efficacy of communication skills training courses in oncology: a systematic review and meta-analysis. Ann Oncol 2010; 22 (5): 1030-40. 